\title{
Two-spacecraft reconstruction of a magnetic cloud and comparison to its solar source
}

\author{
C. Möstl ${ }^{1,2}$, C. Miklenic ${ }^{1}$, C. J. Farrugia ${ }^{3}$, M. Temmer ${ }^{2,4}$, A. Veronig ${ }^{1}$, A. B. Galvin ${ }^{3}$, B. Vršnak ${ }^{4}$, and H. K. Biernat ${ }^{1,2}$ \\ ${ }^{1}$ Institute for Physics, University of Graz, Universitätsplatz 5, 8010 Graz, Austria \\ ${ }^{2}$ Space Research Institute, Austrian Academy of Sciences, Schmiedlstrasse 6, 8042 Graz, Austria \\ ${ }^{3}$ Space Science Center and Dept. of Physics, University of New Hampshire, Durham, NH 03824, USA \\ ${ }^{4}$ Hvar Observatory, Faculty of Geodesy, University of Zagreb, Kačićeva 26, 10000 Zagreb, Croatia
}

Received: 11 September 2007 - Revised: 21 November 2007 - Accepted: 13 February 2008 - Published: 15 October 2008

\begin{abstract}
This paper compares properties of the source region with those inferred from satellite observations near Earth of the magnetic cloud which reached $1 \mathrm{AU}$ on 20 November 2003. We use observations from space missions SOHO and TRACE together with ground-based data to study the magnetic structure of the active region NOAA 10501 containing a highly curved filament, and determine the reconnection rates and fluxes in an M4 flare on 18 November 2003 which is associated with a fast halo CME. This event has been linked before to the magnetic cloud on 20 November 2003. We model the near-Earth observations with the Grad-Shafranov reconstruction technique using a novel approach in which we optimize the results with two-spacecraft measurements of the solar wind plasma and magnetic field made by ACE and WIND. The two probes were separated by hundreds of Earth radii. They pass through the axis of the cloud which is inclined -50 degree to the ecliptic. The magnetic cloud orientation at $1 \mathrm{AU}$ is consistent with an encounter with the heliospheric current sheet. We estimate that $50 \%$ of its poloidal flux has been lost through reconnection in interplanetary space. By comparing the flare ribbon flux with the original cloud fluxes we infer a flux rope formation during the eruption, though uncertainties are still significant. The multi-spacecraft Grad-Shafranov method opens new vistas in probing of the spatial structure of magnetic clouds in STEREO-WIND/ACE coordinated studies.
\end{abstract}

Keywords. Interplanetary physics (Interplanetary magnetic fields) - Solar physics, astrophysics, and astronomy (Flares and mass ejections) - Space plasma physics (Magnetic reconnection)

Correspondence to: C. Möstl

(moestlch@stud.uni-graz.at)

\section{Introduction}

The interplanetary coronal mass ejection (ICME) containing a magnetic cloud (MC) on 20 November 2003 elicited one of the strongest geomagnetic storms of solar cycle 23 (19952006 ) with a minimum $D_{s t}$ value of $-422 \mathrm{nT}$. (For a discussion of storm strength see Bothmer and Zhukov, 2006). Magnetic clouds are defined by a strong magnetic field which rotates smoothly through a large angle in a low proton temperature plasma (Burlaga et al., 1981). They are modeled as helical flux ropes (Burlaga, 1988). Relating them to their solar progenitors has been discussed mainly for quiescent filament eruptions, and good correspondences between orientation and structure have been found (Marubashi, 1986; Bothmer and Schwenn, 1998; Zhao and Hoeksema, 1998; Yurchyshyn et al., 2001; Bothmer, 2003). For active regions (ARs), Leamon et al. (2004) found that integrated fluxes in active regions and in their associated magnetic clouds are comparable. The amount of twist (a quantity proportional to the number of field line turns) is larger in MCs than in the ARs and there is no sign or amplitude relationship between them, hinting that magnetic clouds are formed by magnetic reconnection between the AR and their larger-scale surroundings. For nine events, Qiu et al. (2007) found a close correspondence over several orders of magnitude between magnetic fluxes inferred from two-ribbon flares and the fluxes in associated MCs. Several recent case studies have associated magnetic cloud fluxes with their associated source regions and/or coronal dimming flux (Mandrini et al., 2005; Attrill et al., 2006; Yurchyshyn et al., 2006; Longcope et al., 2007).

Cremades and Bothmer (2004) emphasize that the axis orientation of the erupting flux rope at the Sun is controlled by the active region structure and the ambient corona. Crooker (2000) pointed out that MC axis orientations tend to be aligned with the heliomagnetic equator, for which

Published by Copernicus Publications on behalf of the European Geosciences Union. 


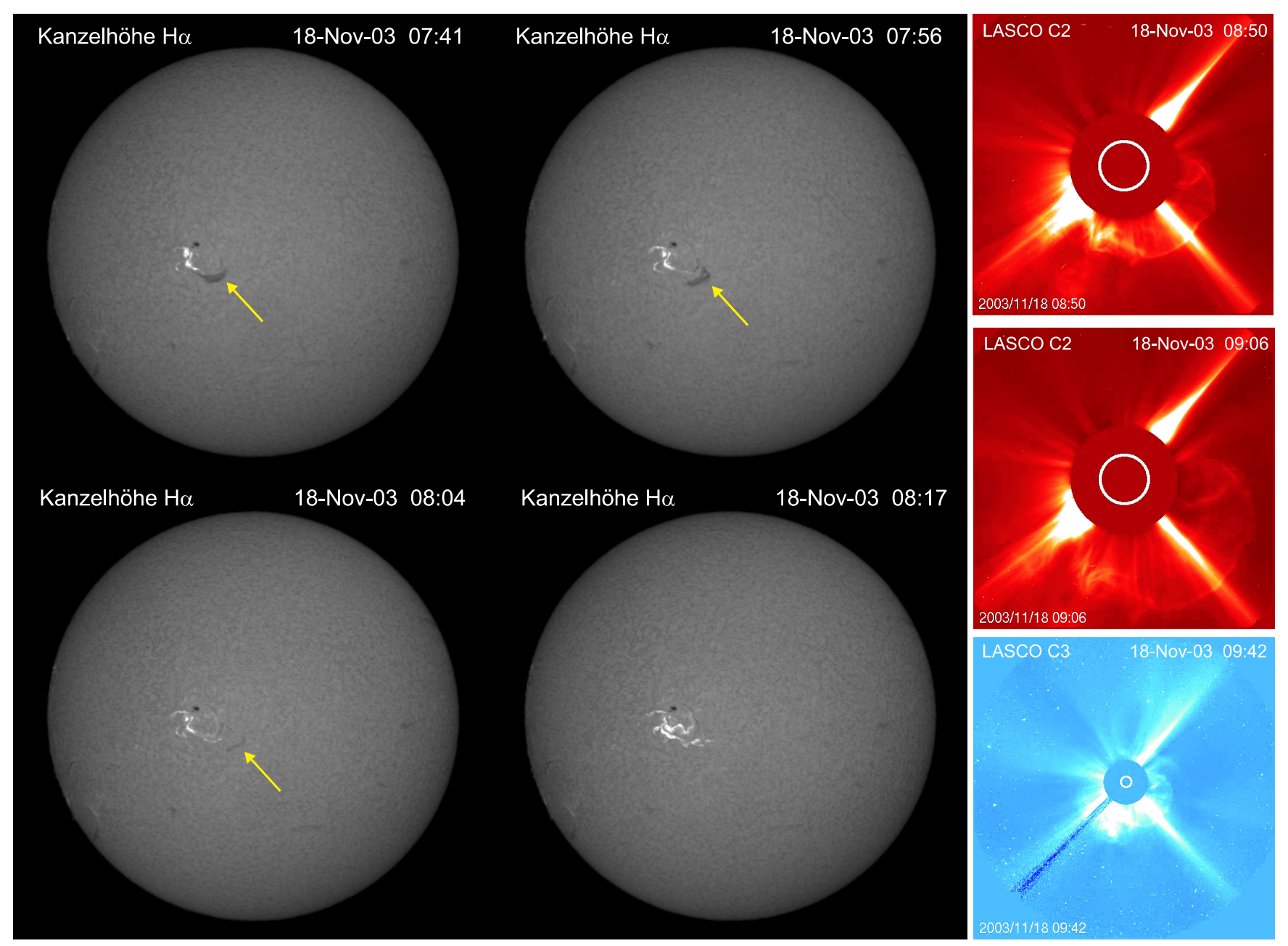

Fig. 1. Left 4 panels: H $\alpha$ filtergrams from the Kanzelhöhe Solar Observatory (Austria) showing the global flare evolution and the erupting filament (indicated by arrows). Right 3 panels: Temporal evolution of the associated CME in SOHO/LASCO C2 and C3.

observational support was invoked (Mulligan et al., 1998). Yurchyshyn et al. (2007) find for $2 / 3$ of 25 events studied a good correspondence between the LASCO halo CME tilt angle and the $\mathrm{MC}$ orientation angle. The orientation is of central importance because MCs are sources of long duration interplanetary (IP) southward magnetic fields which depends on the axis orientation (Zhao and Hoeksema, 1998). MCs are a major cause of strong geomagnetic storms (e.g. Gonzalez and Tsurutani, 1987).

Recent reviews on the many open questions regarding these connections were given by Crooker and Horbury (2006) and Démoulin (2007). Such studies are expected to enhance our ability to forecast space weather (see for instance Siscoe and Schwenn, 2006) and to improve our understanding of solar-terrestrial relations in general. The aim of this paper is to apply an advanced methodology to study the solar M4 flare and the corresponding halo CME on 18 November 2003 and ICME/MC observed near Earth on 20 November 2003, and search for quantitative links in orien- tations and magnetic fluxes. We start with the solar observations of the flare, filament and CME in Sect. 2. We then reconstruct the corresponding magnetic cloud in Sect. 3 and compare its characteristics with the solar source in Sect. 4.

\section{Solar observations}

\subsection{Overview of the 18 November 2003 flare/CME event}

The 20 November ICME has been associated with the halo CME on 18 November 08:50 UT, which originated from AR 10501 (Gopalswamy et al., 2005). The halo CME was a fast one, propagating in the LASCO field of view with a linear speed of $\sim 1660 \mathrm{~km} \mathrm{~s}^{-1}$ (from the SOHO/LASCO CME catalog ${ }^{1}$ ), but decelerated down to $\sim 740 \mathrm{~km} / \mathrm{s}$ by the time it reached Earth. It was discussed in detail by Gopalswamy et al. (2005). The CME was associated with the 18 November

\footnotetext{
${ }^{1}$ http://cdaw.gsfc.nasa.gov/CME_list
} 

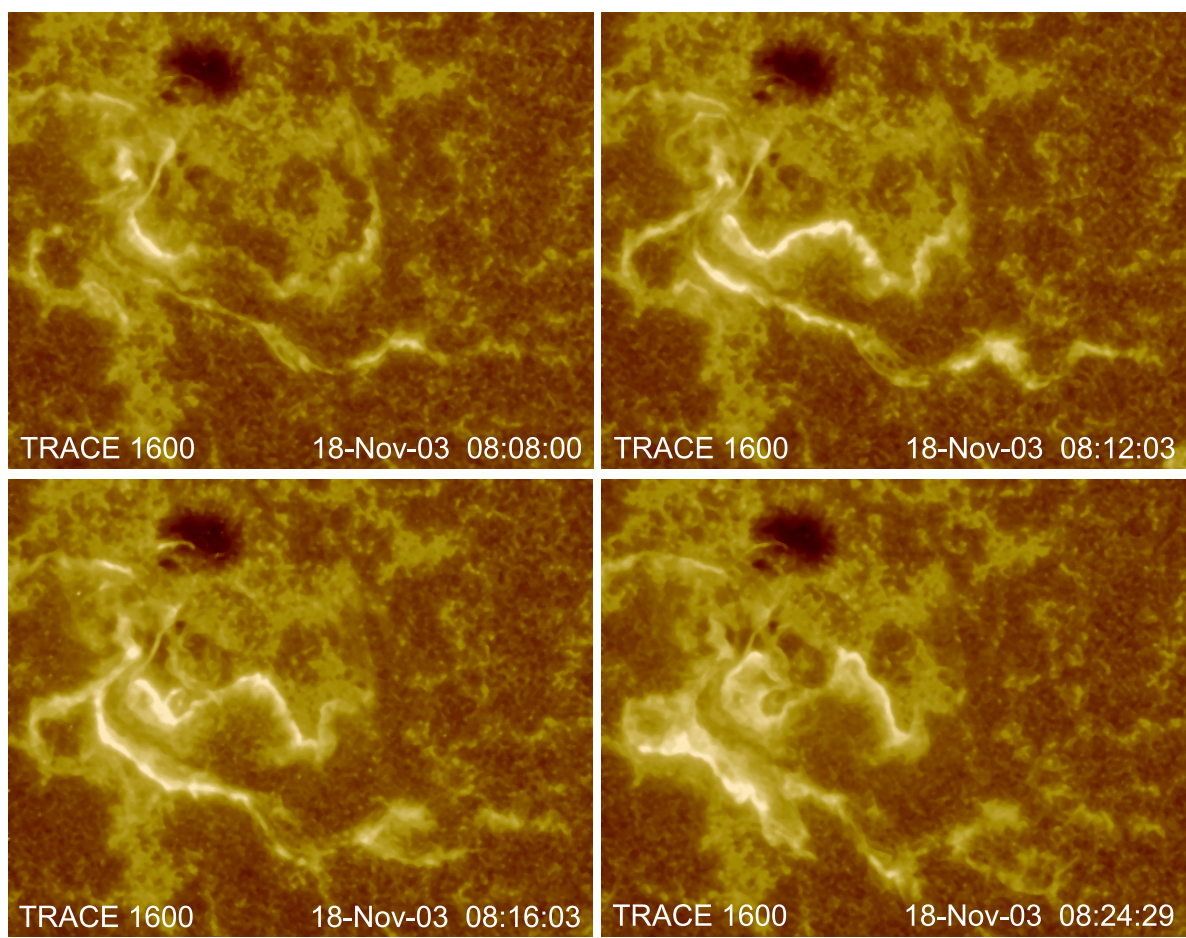

Fig. 2. Temporal evolution of the flare ribbons during the impulsive phase in TRACE UV. Solar north is up, west is to the right. FOV: $320^{\prime \prime} \times 250^{\prime \prime}$.

GOES M3.9/2N flare event, which occurred in the vicinity of AR 10501 at S02E18. The Kanzelhöhe filtergrams at $\mathrm{H} \alpha-0.3 \AA$ in Fig. 1 show the location and evolution of the flaring region on the solar disk. There is a dark U-shaped filament (arrowed), which rises slowly between 07:30 UT (beginning of observations) and 08:00 UT. Around 08:00 UT there is a rapid eruption in a south-west direction, and by 08:08 UT the filament has vanished from the $\mathrm{H} \alpha$ filtergrams. The panels on the right of Fig. 1 show the temporal evolution of the associated CME, whose most prominent front is also south-west bound, though, in fact, it was a halo CME (cf. LASCO C3 image in Fig. 1). Figure 2 shows the temporal evolution of the flare in the $1600 \AA$ UV channel from the Transition Region and Coronal Explorer (TRACE, Handy et al., 1999), with two bright, separating flare ribbons. (A movie of the flare is provided in the online material of Miklenic et al., $2007^{2}$.)

\subsection{Magnetic configuration and orientation of the filament}

CMEs as observed in white-light often exhibit a three-part structure, consisting of a bright front followed by a darker region (cavity) and a bright core. This bright core is thought to be the counterpart of an eruptive prominence/filament (Illing and Hundhausen, 1985) and within this core helical patterns

\footnotetext{
${ }^{2}$ http://www.aanda.org/articles/aa/full/2007/02/aa5751-06/ 5751mov1.mov
}

are often present, possibly indicating an underlying flux rope (see e.g. Chen et al., 1997). Filaments are believed to be embedded in flux ropes, whose magnetic imprints, such as magnetic tilt angle (with respect to the solar equator) and twist, are assumed to be preserved during the eruption (e.g. Bothmer and Schwenn, 1998; Yurchyshyn et al., 2001). The tilt angle may be altered near the Sun through interaction with the ambient corona (Cremades and Bothmer, 2004), by the helical kink instability (e.g. Fan and Gibson, 2003; Rust and LaBonte, 2005) or the overlying coronal field (Crooker, 2000). As shown in Fig. 1, the direction of the filament eruption is well correlated with that of the CME.

Figure 3 shows the evolution of the filament from 15 November until 18 November 2003, using $\mathrm{H} \alpha$ images from the solar observatories Big Bear (BBSO), Mauna Loa (MLSO), and Kanzelhöhe (KSO). The high-resolution BBSO observations show that this filament actually consists of two separate filaments, namely, the U-shaped filament which erupts at 08:00 UT on 18 November, as discussed above, and in the north-west a smaller filament which does not take part in the eruption (see also bottom panel in Fig. 4 and Fig. 5). The TRACE 171 A post-flare loops can be seen over the southern and apex parts of the filament (Fig. 5). This filament and the associated post-flare loops have an inclination angle with respect to the solar equator in the range from $\sim 35^{\circ}$ (bright southern loops) to $\sim-25^{\circ}$ (weaker post-flare arcade associated with the filament apex). In the following, 

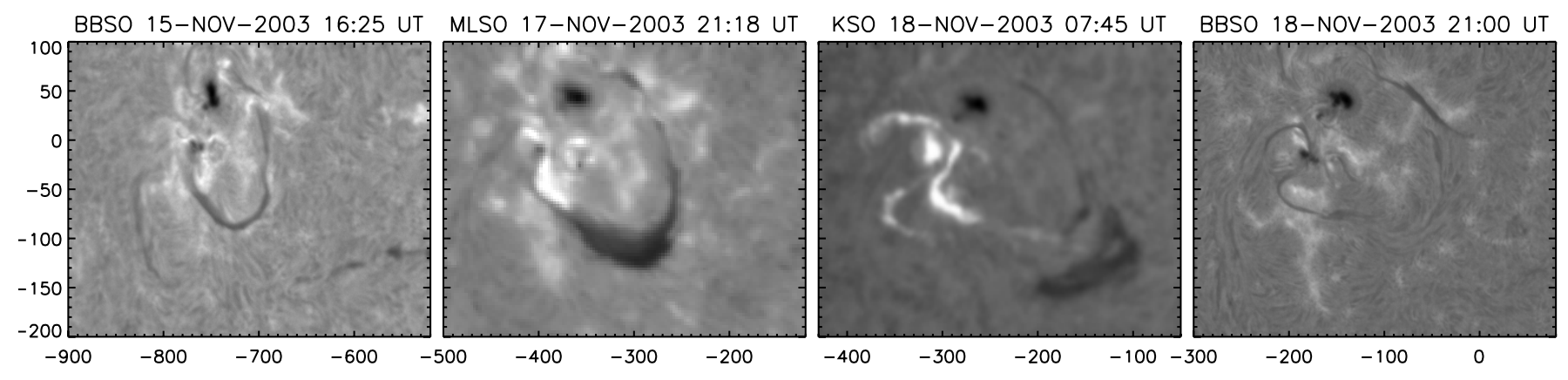

Fig. 3. Evolution of the filament from 15 November until 18 November using H $\alpha$ observations from BBSO, MLSO, KSO, BBSO (left to right). When the active region is close to the limb (left panel, BBSO) the two separate filaments are visible, with the northern filament located at $(-710,60)$.

we assume a $2.5 \mathrm{D}$ situation where a sheared arcade undergoes reconnection and creates a helical flux rope (see Figs. 1 and 3 in Démoulin et al., 1996), containing both poloidal as well as axial magnetic field components. The poloidal magnetic field at the leading edge of an erupting flux rope can be obtained from the underlying magnetic field provided by the MDI/SOI instrument (Scherrer et al., 1995) on board the Solar and Heliospheric Observatory (SOHO), see top panel of Fig. 4. Locations of conjugate HXR footpoints nonperpendicular to the neutral line may refer to the shear of a magnetic arcade and can be used to derive the orientation of the axial field. However, for this event, the orientation of the axial field cannot be derived in a straightforward manner through footpoint-shearing. The post-flare loops and the conjugate footpoints of the first hard X-ray (HXR) emission are oriented roughly perpendicular to the local neutral line and thus gives ambiguous results.

We now discuss briefly other work on this issue. Gopalswamy et al. (2005) derived a right-handed twist from the magnetic field polarity of the filament's footpoints. In addition, Yurchyshyn et al. (2005) found that the line connecting these footpoints has a similar orientation as the MC axis at $1 \mathrm{AU}$ (see Sect. 3.3). These authors did not consider the two separated filaments. Restricting the magnetic field to the erupting filament only and applying the same method as Gopalswamy et al. (2005), a negative twist would be the result. Yurchyshyn et al. (2005) obtained a positive twist of the post-flare loops using a linear force-free field model. To keep our discussion limited we will assume further an axial field pointing to the east, which, together with the poloidal field, implies (Fig. 4) a right-handed flux rope, inline with the magnetic cloud observations at $1 \mathrm{AU}$. We note that the solar observations are ambiguous, and that in an active region a left-handed flux rope in the low corona may also be associated with a right-handed MC, as in some cases reported by Leamon et al. (2004). This will be further discussed in Sect. 4.2.

\subsection{Flare reconnected magnetic flux}

Magnetic reconnection causes a topological restructuring of the field and allows the release of stored magnetic energy to heat plasma, drive plasma flows, and accelerate particles up to relativistic energies. Oppositely directed magnetic field lines enter the tiny diffusion region in the corona, where they reconnect. In the case of a sheared arcade, the reconnected flux is divided into (a) a loop disconnected from the Sun which is propelled out into interplanetary space, and (b) lower lying field lines collapsing back onto the solar surface (e.g. Démoulin et al., 1996). Since magnetic flux is conserved, the amount of flux entering and leaving the reconnection region is the same, and the lower lying flux is equal to the flux which escaped into interplanetary space. Thus, the reconnected flux can be determined from chromospheric/photospheric observations, such as $\mathrm{H} \alpha / \mathrm{UV}$ image sequences, which show the flare brightenings, and photospheric line-of-sight magnetograms. Derived positive and negative fluxes should be equal since equal amounts of positive and negative magnetic flux participate in reconnection.

The reconnected magnetic flux $\Phi_{r, t}$ at a particular time $t$

$\Phi_{r, t}=\int_{t_{0}}^{t} B_{n} d a$,

is determined separately for each magnetic polarity domain (Forbes and Lin, 2000). Here, $B_{n}$ is the magnetic field strength component perpendicular to the solar surface in the newly brightened area $d a$ that is swept by the flare ribbons. The reconnection rate, $\dot{\Phi}$, can be obtained by taking the time derivative of Eq. (1), and it can be compared with the observed hard X-ray (HXR) emission. This bremsstrahlungemission is generated by fast electrons, which have been accelerated at the reconnection site and which deposit their energy at the chromosphere. Since the intensity of HXR emission is proportional to the number of accelerated electrons, and this number in turn is proportional to the rate at which energy is released at the reconnection site, HXR emission is 

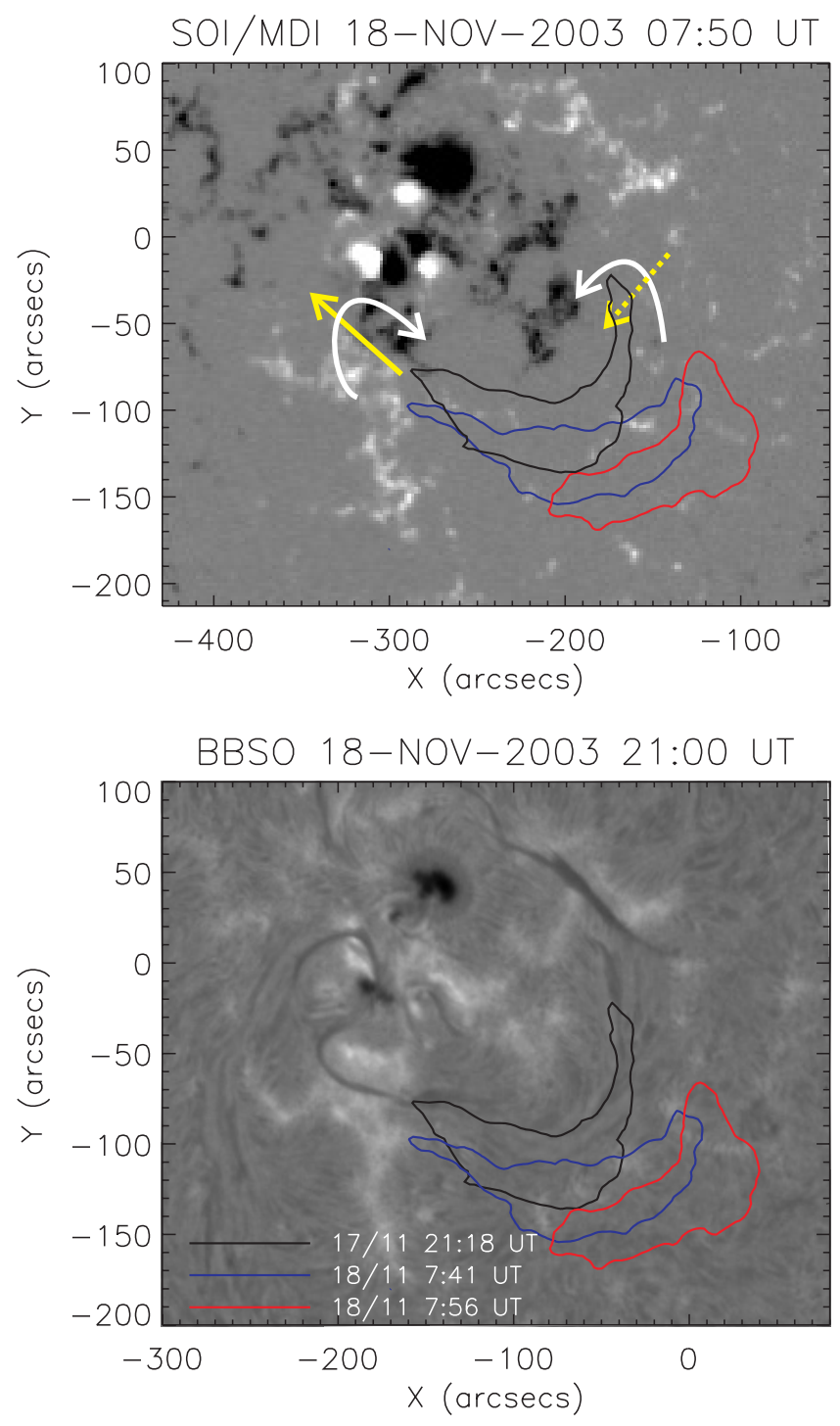

Fig. 4. Top: MDI line-of-sight magnetogram of the active region (white - positive polarity; black - negative polarity) overlaid with contour lines of the U-shaped filament as seen in $\mathrm{H} \alpha$ from MLSO the day before (black line) and KSO during the eruption (blue and red lines). White and yellow arrows indicate the leading poloidal and the axial field direction, respectively. Bottom: BBSO $\mathrm{H} \alpha$ image after the eruption overlaid with the same contour lines.

considered to be proportional to the energy release rate in a solar flare (Hudson, 1991), and can therefore be used as a proxy for the reconnection rate. Both derived reconnection rate and observed HXR emission should evolve similarly in time.

A TRACE $1600 \AA$ image sequence with a cadence of $\sim 23 \mathrm{~s}$ was used to determine the newly brightened area (NBA) in an image compared to the preceding images (cf. Fig. 2). The TRACE observations covered a good portion of the impulsive phase, i.e. the period where signatures of

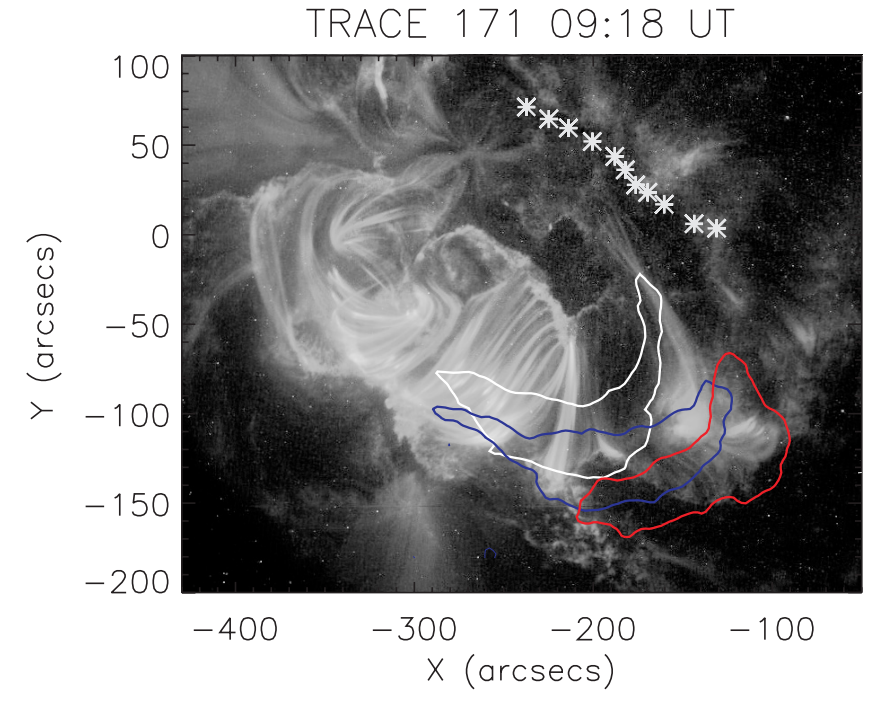

Fig. 5. TRACE $171 \AA$ post-flare arcade at 09:18 UT overlaid with the same filament contour lines as described in Fig. 4 (white is the MLSO contour line). The white asterisks give the location of the northern filament which did not erupt.

high-energy particles are observed in RHESSI (Reuven Ramaty High Energy Solar Spectroscopic Imager; Lin et al., 2002) nonthermal HXR emission (see Fig. 7). The longitudinal, photospheric magnetic field was derived from a full-disk magnetogram before flare onset provided by the SOHO/MDI instrument. The MDI and TRACE data sets were differentially rotated to the same time (07:50 UT) and co-registered.

According to Eq. (1), the reconnected flux at time $t$ is the newly brightened area times the magnetic field strength at this area. Since each area unit in an image is equal to 1 pixel, it is sufficient to add up the magnetic field strength values at each newly brightened pixel in an image and to convert 'square pixels' to $\mathrm{km}^{2}$ to get the reconnected positive and negative magnetic flux at time $t$. Positive and negative fluxes should be equal, since the magnetic flux, which participates in the reconnection process, originates in equal amounts from the positive and negative magnetic polarity domains. The total reconnected flux $\Phi_{r}$ is the sum of all fluxes in all pixels that brightened during any period of the flare. Further details on the methods that were used to determine the reconnected magnetic flux can be found in Qiu et al. (2004) and Miklenic et al. (2007).

The upper panel of Fig. 6 is a snapshot of the flaring region near the end of the impulsive phase (cf. Fig. 2). In the middle panel, all TRACE pixels that brightened during any period of the analyzed time interval of the flare are superposed on that TRACE image. In the bottom panel, the contours of these flare pixels have been superposed on the MDI magnetogram. The northern ribbon sweeps the negative polarity domain (blue), whereas the southern ribbon goes through the positive domain (red). 

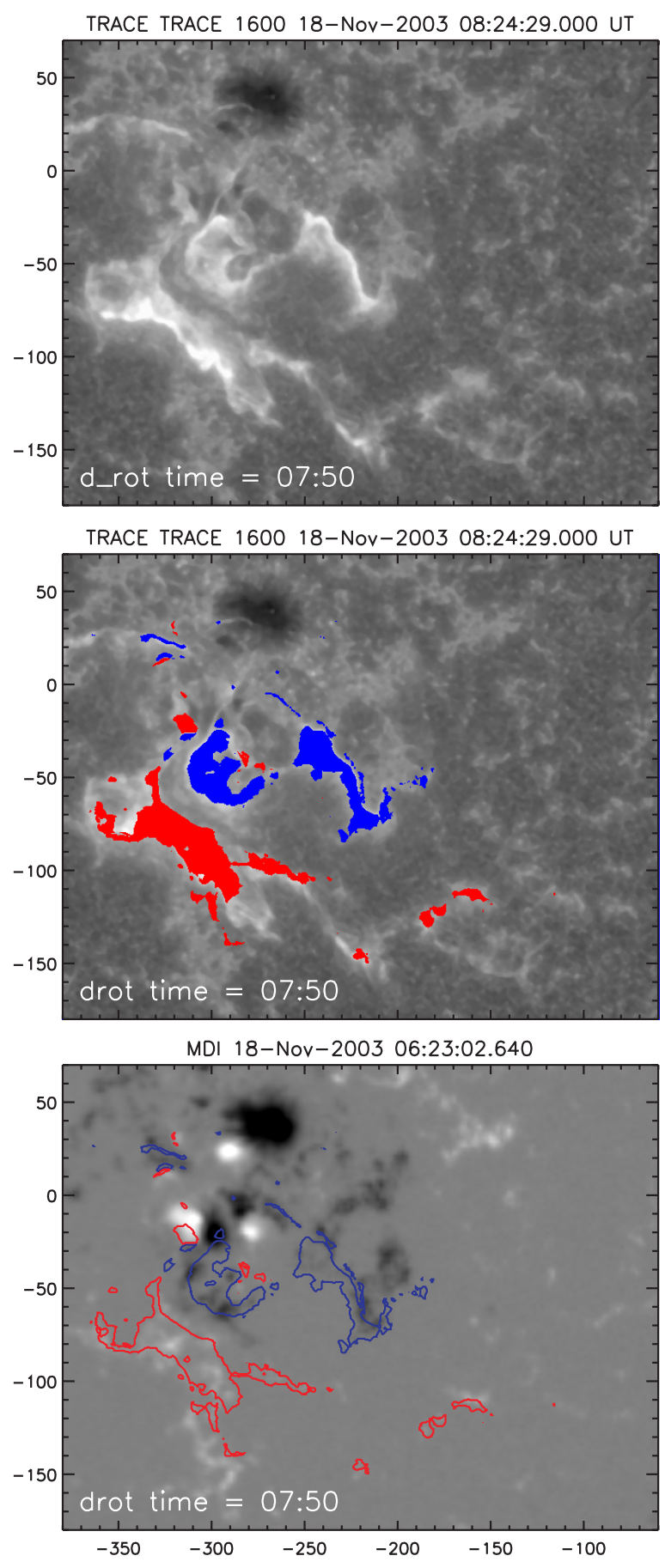

Fig. 6. Upper panel: TRACE $1600 \AA$ flare ribbons near the end of the impulsive phase. Middle panel: Blue/red pixels mark all TRACE pixels that brightened during any period of the analyzed time interval of the flare, where blue areas refer to negative and red areas to positive magnetic polarities of the underlying photospheric magnetic field. Bottom panel: Contours of the total flare area superposed on a MDI magnetogram, where white/black patches represent the positive/negative magnetic polarity domains.

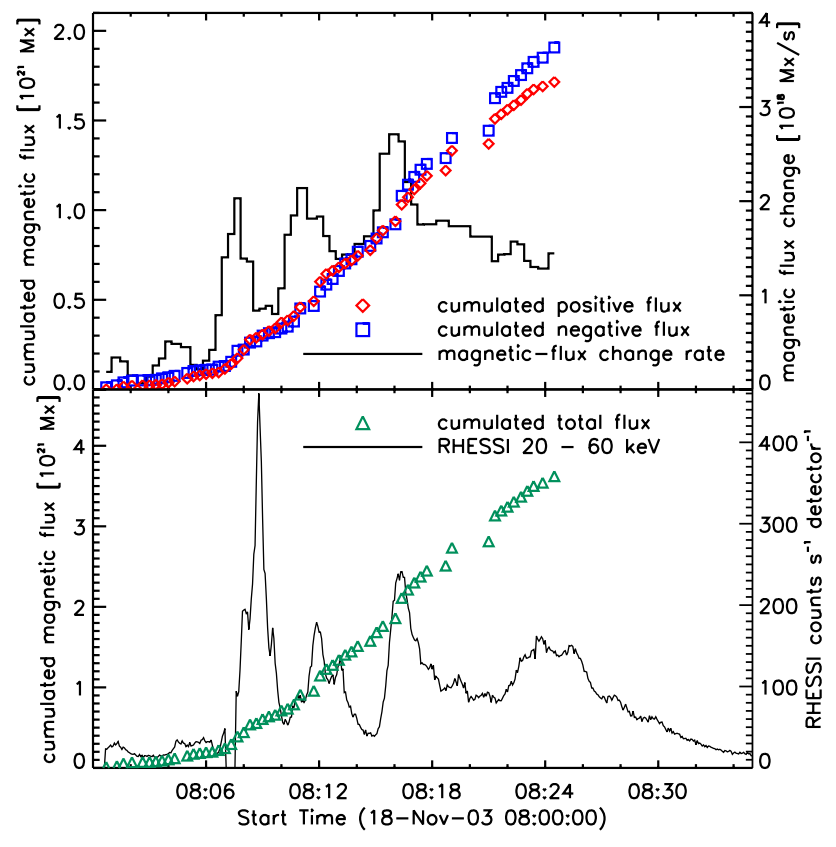

Fig. 7. Upper panel: Reconnection rate and cumulated positive and negative magnetic flux derived during the flare impulsive phase. Lower panel: Cumulated total magnetic flux and RHESSI 20$60 \mathrm{keV}$ HXR time profile.

The upper panel of Fig. 7 shows the derived reconnection rate along with the accumulated positive and negative magnetic flux during the flare impulsive phase. Although near the end of the impulsive phase the flux profiles start to diverge, before this time they are very similar, as is theoretically expected (correlation coefficient over the entire time interval $=0.99$ ). Up to the end of the analyzed time interval, $\Phi^{+}=1.7 \times 10^{21} \mathrm{Mx}$ of positive and $\Phi^{-}=-1.9 \times 10^{21} \mathrm{Mx}$ of negative magnetic flux, respectively, have been reconnected, indicating that indeed equal amounts are involved. In the lower panel of Fig. 7, the total reconnected magnetic flux $\Phi_{r}$ is plotted along with the RHESSI $20-60 \mathrm{keV}$ light curve, which is nonthermal bremsstrahlung produced by fast electrons with energies $\geq 20 \mathrm{keV}$. RHESSI observed four main peaks during the impulsive phase. A visual comparison of the RHESSI profile with the derived reconnection rate in the upper panel shows that the three peaks that were observed in the HXR flux also appear clearly in the reconnection rate. Furthermore, the reconnected magnetic flux profile steepens at the beginning of each HXR burst, which means that more magnetic flux is reconnected during the HXR bursts than in the time intervals between them. This indicates that pulses in the magnetic flux transfer rate are associated with bursts of accelerated electrons. The overall magnetic flux that has been reconnected during the analyzed time interval adds up to $\Phi_{r}=3.6 \times 10^{21} \mathrm{Mx}$. The actual value might be somewhat higher, since the very end of the flare impulsive phase, as indicated by the RHESSI HXR flux, was not covered by 


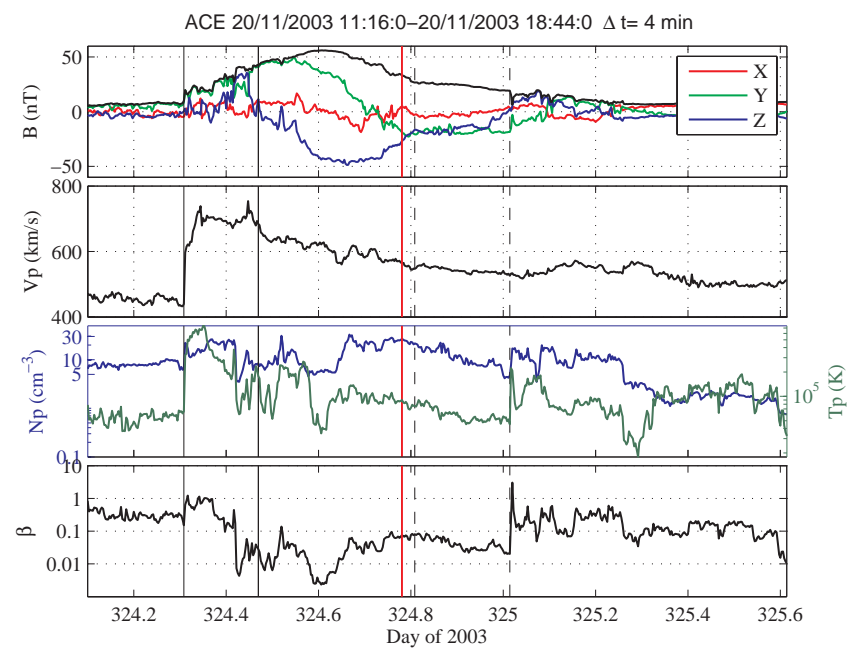

Fig. 8. Data from the ACE spacecraft, from top to bottom: Magnetic field components (coloured as indicated) and absolute value (black line), proton bulk velocity, proton number density and temperature as well as proton $\beta$ (ratio of plasma to magnetic pressure). The first vertical solid line indicates the shock, and the second and third the final GS-reconstruction interval. Dashed vertical lines are the boundaries of the back region.

TRACE observations, and since the applied intensity threshold does not identify faint flare pixels.

\section{Distant WIND-ACE observations at 1 AU}

\subsection{Data}

We now consider the near-Earth observations of the ICME. In Figs. 8 and 9, in situ solar wind observations by ACE and WIND are shown for 20-21 November 2003. The WIND magnetic field data are from the MFI (Lepping et al., 1995) and the plasma data from the SWE (Ogilvie et al., 1995) instrument. ACE data were acquired by the MAG (magnetic field, Smith et al., 1998) and SWEPAM (plasma, McComas et al., 1998) instruments. Both data sets were linearly interpolated to a 4-min time resolution. The average spacecraft positions in GSE were [240, 26, -9] $R_{E}$ for ACE at L1 and [-212, $-38,-13] R_{E}$ for WIND, far down the tail direction and on the dawn side of the magnetosphere. The shock reached ACE at 07:24 UT, 20 November and WIND about $1.07 \mathrm{~h}$ later at 08:28 UT. The initial time interval of the MC at ACE was chosen where the magnetic field strength is above average, the field rotates smoothly and plasma $\beta$ is well below unity (Burlaga et al., 1981). The interval at WIND follows from a time shift given by the shock arrival time. The boundaries are then further adjusted in the course of our reconstruction technique such that the vector potential $A$ has approximately the same value at the back as at the

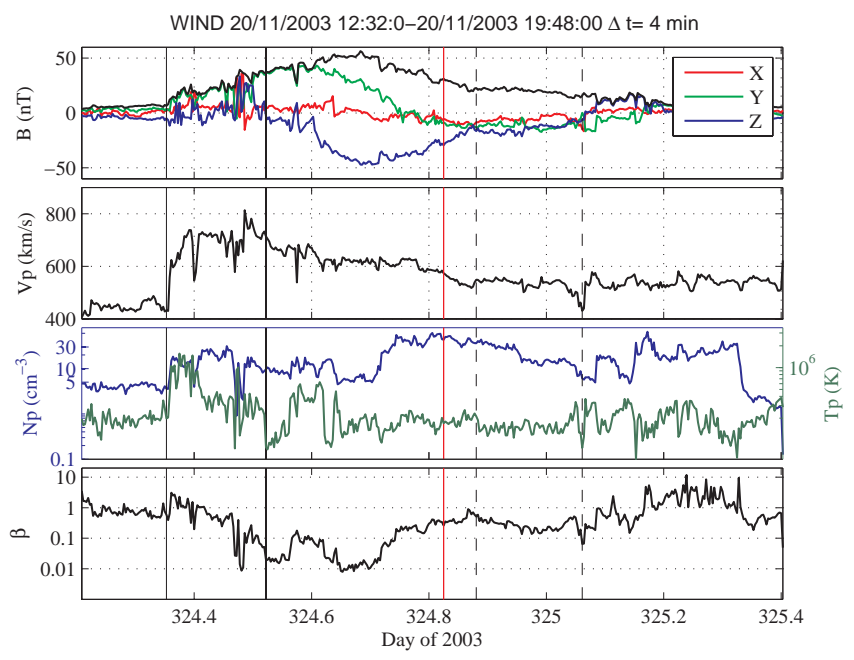

Fig. 9. Data from the WIND spacecraft. Same format as Fig. 8 .

front (Hu et al., 2004). We find a duration of the MC from 11:16 UT, 20 November to 18:44 UT, 20 November at ACE and 12:20 UT, 20 November to 19:48 UT, 20 November at WIND. Both final reconstruction intervals have the same duration of $7 \mathrm{~h}$ and $28 \mathrm{~min}$. Inside the $\mathrm{MC}$ interval (solid lines), the plasma bulk velocity decreases, indicating an ongoing radial MC expansion (e.g. Farrugia et al., 1993). We have also marked the boundaries (dashed lines) of the MCs back region, to be discussed in Sect. 4.1.

\subsection{Grad-Shafranov reconstruction technique}

The GS technique (Hau and Sonnerup, 1999) is a versatile tool to reconstruct space plasma structures possessing an invariant direction. Hu and Sonnerup (2002) first applied this technique to magnetic clouds. The structures are treated as time-independent and we work in the co-moving deHoffmann-Teller frame (Khrabrov and Sonnerup, 1998) with constant velocity $\boldsymbol{V}_{H T}$. It allows us to recover a $21 / 2$ dimensional cross section of the MC magnetic structure in a plane perpendicular to the invariant axis by integrating away from the spacecraft observations (trajectory) which are used as initial values, solving the Grad-Shafranov equation:

$$
\frac{\partial^{2} A}{\partial x^{2}}+\frac{\partial^{2} A}{\partial y^{2}}=-\mu_{0} \frac{d P_{t}(A)}{d A} \equiv-\mu_{0} \frac{d\left(p+B_{z}^{2} / 8 \pi\right)}{d A} .
$$

Due to time-independency, time intervals can be directly converted into spatial distances with $d x=-\boldsymbol{V}_{H T} \cdot \hat{\boldsymbol{x}} d t$. The vector potential $A$ can then be calculated as

$A(x, 0)=-\int_{0}^{x} B_{y}(x, 0) d x$.

The single-spacecraft implementation of the technique uses the fact that the pressure transverse to the invariant direction 


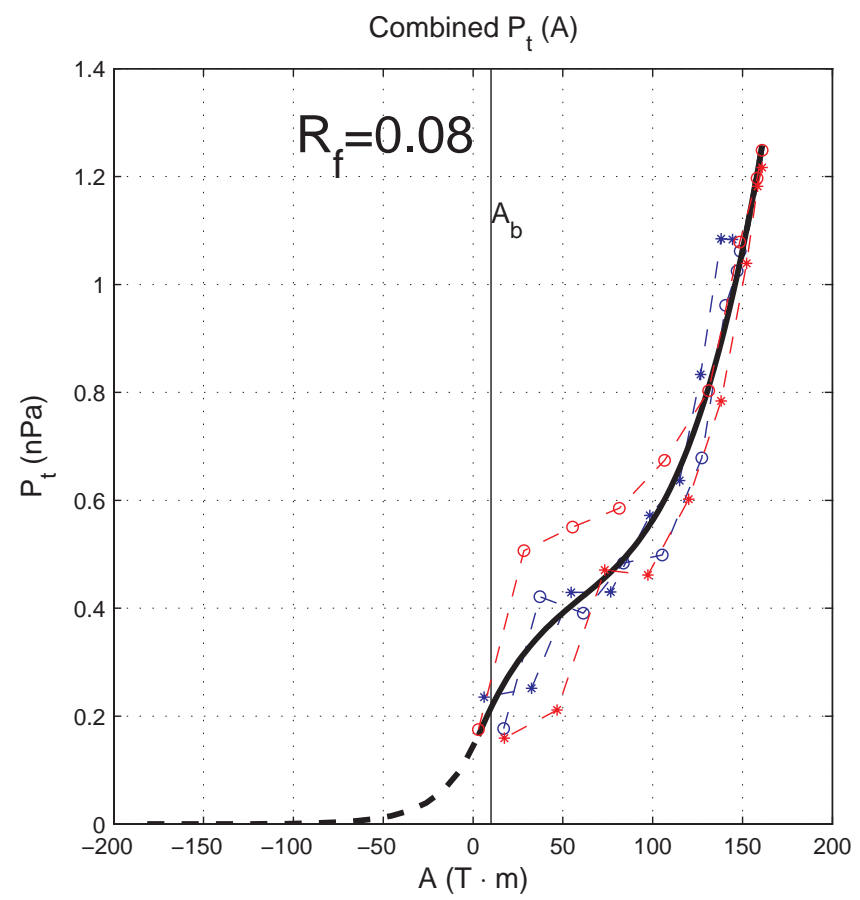

Fig. 10. Combined $P_{t}(A)$ plot (red ACE, blue WIND) with residue $R_{f}=0.08$. A third order polynomial is fitted (solid black) with an exponential tail (dashed black). Scattered points are the observations - circles stand for the first half and stars for the second half of the event. The vertical line drawn at a value $A_{b}=10 \mathrm{~T} \mathrm{~m}$ corresponds to the white contour line in Fig. 11.

$P_{t}(A)$ must be single valued along a spacecraft trajectory to find the correct orientation of the clouds axis (Hu and Sonnerup, 2002). As discussed in Möstl et al. $(2008)^{3}$ we have adapted the multi-spacecraft approach by Sonnerup et al. (2004) and Hasegawa et al. (2005, 2006).

\subsection{Reconstruction of the 20 November 2003 MC}

Here we summarize the two-spacecraft reconstruction method. The deHoffmann-Teller analysis of the combined set of magnetic field and plasma velocities yields $V_{H T}=615 \mathrm{~km} / \mathrm{s}$, a correlation coefficient of 0.9969 and a Walén slope of $w=0.0078$. The combined plots of $P_{t}(A)$ (Fig. 10) and $B_{z}(A)$ (not shown) are fitted by a third order polynomial with exponential tails. The quality of this fit is measured by the fitting residue $R_{f}=0.08$ (for a definition see $\mathrm{Hu}$ et al., 2004). With this relation defining the right-hand side of the Grad-Shafranov Eq. (2), two independent magnetic field maps, one for ACE and one for WIND, are produced by integrating away from the spacecraft measurements along their respective trajectory used as initial values. These are then combined into a composite map using a Gaussian window function, as described in Hasegawa et al. (2005). A correlation coefficient $c c$ between the magnetic field compo-

\footnotetext{
${ }^{3}$ Möstl, C., Farrugia, C. J., Biernat, H. K., et al.: in preparation, 2008.
}

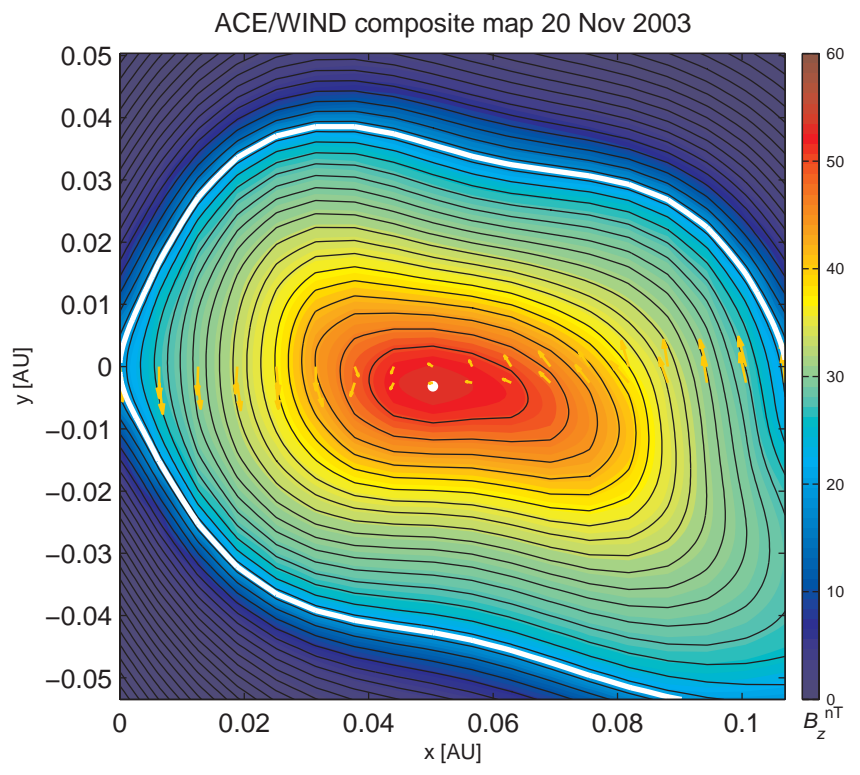

Fig. 11. Combined magnetic field map. Contour lines represent transverse magnetic field lines. $B_{z}$ (pointing out of the paper, along the invariant direction) is color coded, with its maximum at the white dot which corresponds to $A_{m}=155 \mathrm{~T} \mathrm{~m}$ (see text). The white contour line is drawn at a value of $A_{b}=10 \mathrm{~T} \mathrm{~m}$. Yellow arrows along the spacecraft trajectory are projections of the measured field vectors onto the $\mathrm{x}$-y plane (upper WIND, lower ACE).

nents predicted by the map at the location of the spacecraft and those actually observed is calculated.

From the dependence of $R_{f}$ and $c c$ on the latitude and longitude of the invariant axis we select the one where a high correlation coefficient is matched by a low value of $R_{f}$, the latter being important to ensure that $P_{t}(A)$ is single valued. Doing this we find the optimal axis at $\theta=-50 \pm 4^{\circ}$ and $\phi=80 \pm 10^{\circ}$ in GSE (Angle $\theta$ is the inclination to the ecliptic and $\phi$ is measured from the sunward direction towards east). There, $R_{f}=0.08$ and $c c=0.9883$. The impact parameter for WIND is $3 \times 10^{-3}$ AU and for ACE $5 \times 10^{-4}$ AU. By comparison, from minimum variance analysis (MVA) of the same time interval we obtain $\theta=-55^{\circ}$. MVA, force-free fitting (FF) and GS methods have been applied by other authors. We now cite these results in terms of the axis inclination $\theta$ : (i) $-49^{\circ}$ (GS, Yurchyshyn et al., 2005) (ii) $-73^{\circ}$ (FF, Gopalswamy et al., 2005) (iii) $-87^{\circ}$ (FF, Lynch et al., 2005) (iv) $-71^{\circ}$ (MVA, Huttunen et al., 2005). We note that not all intervals examined were the same. Trendwise, for the short interval (Yurchyshyn et al., 2005, and this study) the inclinations are less.

The MC axis is highly inclined to the ecliptic and points towards east. The longitude of the axis implies that the spacecraft encounter the apex of the flux rope. The MC is right-handed, and the combined magnetic field map is shown in Fig. 11. The reconstruction 
coordinate system is $\hat{\boldsymbol{x}}^{\prime}=[0.9936,-0.0861,0.0736]$, $\hat{\boldsymbol{y}}^{\prime}=[-0.0193,-0.7693,-0.6386], \quad \hat{z}^{\prime}=[0.1116, \quad 0.6330$, $-0.7660]$ in GSE. Note that the $\hat{\boldsymbol{y}}^{\prime}$-axis points south of the ecliptic. From Fig. 11 it can be seen that the field lines are not circular, but resemble rather ellipses elongated especially in the $\hat{\boldsymbol{x}}^{\prime}$-direction, i.e. along the Sun-Earth line, with an estimated eccentricity of $\sim 2$.

\subsection{Magnetic fluxes}

The toroidal (axial) flux is given by $\Phi_{t}=\iint B_{z} d x d y$, and is determined by the MC cross-section and the axial magnetic field strength. Inside of a boundary set at $A_{b}=10 \mathrm{~T} \mathrm{~m}$ we find a $\Phi_{t}=0.55 \times 10^{21} \mathrm{Mx}$. The poloidal flux is determined by $\Phi_{p}=\left|A_{m}-A_{b}\right| L$, with $A_{m}$ being the vector potential at the MC axis (white dot in Fig. 11), $A_{b}$ the vector potential at the MC boundary (white contour in Fig. 11) and $L$ the length of the MC. With $A_{m}=155 \mathrm{Tm}$ and $A_{b}=10 \mathrm{~T} \mathrm{~m}$ determined from the reconstruction, we find a $\Phi_{p}=2.2 \times 10^{21} \mathrm{Mx} / \mathrm{AU}$. We now discuss the uncertainties in the determination of $\Phi_{t}$ and $\Phi_{p}$.

We calculate a lower limit of the toroidal flux because we use a limited reconstruction domain. It is expected from MHD simulations (e.g. Manchester et al., 2004), theoretical considerations (Russell and Mulligan, 2002) and recent observations (Liu et al., 2006) that the MC transverse size, i.e. perpendicular to the radial and axial directions (usually out of the ecliptic), may be up to an order of magnitude larger compared to its radial size ("flattening" or "pancake shape", Riley and Crooker, 2004). However, "flattening" may not be of importance for this particular MC, because the elliptical cross-section is elongated along the radial direction. Additionally, in Fig. 12 we have plotted results for $\Phi_{t}$ for different boundaries $A_{b}$. Looking from right to left in Fig. 12 we see that $\Phi_{t}$ rises almost linearly with decreasing $A_{b}$. For lower values of $A_{b}$ the slope decreases due to the lower magnetic fields in the outskirts of the cloud. This means that we can assume that the full toroidal flux may not exceed much the value of $\Phi_{t}=0.6 \times 10^{21} \mathrm{Mx}$ that we infer at the very outermost boundary where $A_{b}=0$. Though the area of the flattened part of the cloud may well be much larger, its weak field strengths do not add much more to the axial flux.

We now estimate the poloidal flux. By far the largest uncertainty arises from the unknown length $L$ of the flux rope. There is evidence that both feet of particular MCs were still connected to the Sun (e.g. Farrugia et al., 1993, 2002; Shodhan et al., 2000), though there are no direct observations. Larson et al. (1997) measured $L$ for one event to be $2.5 \mathrm{AU}$. In the ACE electron pitch angle distribution ${ }^{4}$, bidirectional streaming electrons are intermittently visible from 14:00 UT to 18:00 UT 20 November 2003, i.e. in the second half of the MC, suggesting a connection to the Sun. Additionally,

\footnotetext{
${ }^{4}$ http://www.srl.caltech.edu/ACE/ASC/DATA/level3/swepam/ index.html
}

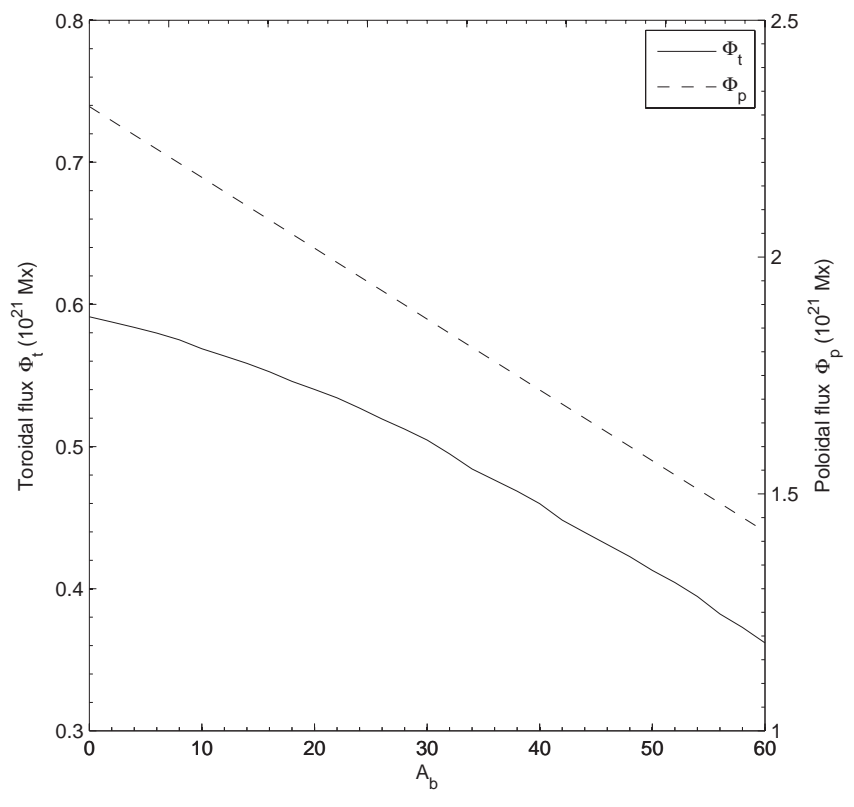

Fig. 12. Fluxes inside different boundaries of the vector potential $A_{b}$, from the inside of the flux rope (right) to the outside (left).

it is not known how well the magnetic structure at the feet of the cloud matches the one we infer at its apex. Farrugia et al. (2005) showed ICME magnetic field scale lengths to be at least 400 Earth radii, using the largest separations attained by near-Earth spacecraft at the time of the study. In the 20 November 2003 magnetic cloud, $\Phi_{p}$ is not influenced by the cross-section because the impact parameter is negligible and we find it also insensitive to small changes in the orientation and boundaries. As seen from the definition and from Fig. 12, $\Phi_{p}$ is inversely proportional to $A_{b}$. Within the boundary $\left(A_{b}=10\right)$ we calculate $\Phi_{p}=2.2 \times 10^{21} \mathrm{Mx}$ per AU. In the view of all these limitations, choosing a plausible length at $1 \mathrm{AU}$ in a range between 0.5 and $2 \mathrm{AU}$ (same as in Qiu et al., 2007) and varying $A_{b}$ between 0 and 20 yields $\Phi_{p}=1.1-4.4 \times 10^{21} \mathrm{Mx}$.

\section{Comparison}

In this section we link the results of Sects. 2 and 3, discuss several possible scenarios to account for discrepancies and try to obtain a coherent picture of what has happened from the time of the flare/CME eruption on the Sun to the arrival of the MC at Earth.

\subsection{Flare reconnection flux and magnetic cloud fluxes}

The part of the reconnection flux which closes down onto the solar surface as inferred from flare ribbon observations is $\Phi_{\bar{r}}=\left(\Phi^{+}+\Phi^{-}\right) / 2$. This is found to be $\Phi_{\bar{r}}=1.8 \times 10^{21} \mathrm{Mx}$ and should be considered as a lower limit. This should be 
compared with the fluxes in the MC as measured at $1 \mathrm{AU}$ : $\Phi_{p}=1.1-4.4 \times 10^{21} \mathrm{Mx}$ and $\Phi_{t}=0.55 \times 10^{21} \mathrm{Mx}$.

We now estimate the original MC flux near the Sun. When the MC is propagating through the IP space, reconnection with the IMF at its boundaries may progressively sweep away magnetic field lines (e.g. McComas et al., 1994). Therefore, we may expect that a significant amount of the original flux (both $\Phi_{t}$ and $\Phi_{p}$ ) has been removed from the MC prior to observations at $1 \mathrm{AU}$ if reconnection has taken place. Farrugia et al. (2001) found a reconnection layer associated with a magnetic cloud, which separated two regions of ejecta material. Many other examples of reconnection related to ejecta have since been found. In the numerical simulations of Schmidt and Cargill (2003) the location of the reconnection site is found to depend on the direction of the MC leading field and the surrounding IMF, and their reconnection rate increases with increasing relative speed between the $\mathrm{MC}$ and the solar wind. While propagating away from the Sun towards the HCS, the 20 November 2003 MC is in the IMF-toward sector $\left(B_{x}>0\right.$, IMF $\left.B_{y}<0\right)$, and the MC leading field is pointing to the north and east $\left(B_{y}>0\right)$. Based on these simulations (Schmidt and Cargill, 2003), we may thus expect that reconnection is taking place from the leading edge to the southern flank of the MC. The speed difference between the sheath plasma $(\sim 730 \mathrm{~km} / \mathrm{s})$ and the downstream solar wind $(\sim 420 \mathrm{~km} / \mathrm{s})$ is also significant. As recently discussed by Dasso et al. (2006, 2007), further evidence for such reconnection is an extended back region of non-rotating smooth magnetic field lines in a low proton $\beta$ plasma. This may be the case for our event as can be seen from Figs. 8 and 9 (the two dashed lines behind each cloud observation delimit this interval). Using Eq. (6) by Dasso et al. (2007) we estimate the time since this reconnection started to $\sim 30 \mathrm{~h}$. We can crudely estimate the flux that was lost since the MC was expelled from the Sun as $\sim 50 \%$. We obtained this by integrating the vector potential $A$ to the back boundary, yielding $A_{b}=-150$, thus $\Phi_{p}$ would be increased by a factor of $2\left(\Phi_{p}=2.2-8.8 \times 10^{21} \mathrm{Mx}\right)$. This is now in very good agreement with the fluxes obtained for the longer interval by forcefree fitting and single-spacecraft GS (Lynch et al., 2005; Qiu et al., 2007), only our physical interpretation is different.

Thus the flux closing down onto the solar surface into the post-flare loops and the original MC poloidal flux fit within a factor of $1-4$, and the MC toroidal flux is an order of magnitude lower. This good agreement between the flare reconnection flux and the MC poloidal flux is not unique to this event and allows to discriminate between a variety of scenarios (see discussion in Qiu et al., 2007). If the helical structure of a flux rope is predominantly formed in situ then $\Phi_{p} \sim \Phi_{\bar{r}}$, while the presence of a substantially twisted pre-existing flux rope implies $\Phi_{p}>\Phi_{\bar{r}}$. Our result suggests that reconnection adds a large amount of poloidal flux during an in situ formation of an erupting flux rope which is then recognized in IP space as a magnetic cloud (cf. Leamon et al., 2004). Either a significant amount of poloidal flux is added to a pre-existing flux rope or the complete MC flux rope is created by this reconnection. However, due to the significant uncertainties arising from present observations, we cannot yet discriminate between these two possibilities.

\subsection{The orientation of the axis}

The spacecraft encounter the MC apex and thus we can exclude projection effects that might weaken the correlation to the solar source orientation. There is a difference in orientation between the magnetic cloud axis in IP space and the associated bright flaring arcade (southern part of the filament) by about $\sim 90^{\circ}$, turning counter-clockwise between Sun and Earth. The orientation of the filament apex differs by only $\sim 30^{\circ}$ with respect to the $\mathrm{MC}$ axis (taking into account the difference between ecliptic north and the solar rotation axis $\sim 7^{\circ}$ ). Since the reconnected flux of the flare and the flux in the MC are in good agreement, we have to assume that the southern part of the erupted filament (i.e. outlined by the bright post-flare arcades, Fig. 5) changed its orientation. The question arises whether this rotation has taken place close to the Sun or while the MC was in the IP medium.

A recent study by Yurchyshyn et al. (2007) revealed that the associated halo CME had an inclination of $\sim-35^{\circ}$ in the LASCO field of view which lies in between the MC axis orientation and the filament orientation at the Sun. The inclination of a halo CME is the angle between the solar equator and the semi-major axis of the elliptical model fitted by Yurchyshyn et al. (2007) to the halo CME's white light signature. From this it may follow that the major change in axis orientation took place already close to the Sun. The helical kink instability is a candidate mechanism for explaining the rotation of the axis in the solar corona (e.g. Fan and Gibson, 2003; Rust and LaBonte, 2005). However, in the present event, the right-handed flux rope turns counter-clockwise, which is at variance with what is expected from this instability, because, by virtue of magnetic helicity conservation, right handed twist turns into positive writhe through a clockwise rotation. There is also no particular evidence from the filament eruption that any winding took place during the liftoff. A small coronal hole is observed to the east of the solar source region which could result in some interaction between a faster stream and the CME and might have constrained the eruption to the south-west, but the possibility that the axis rotated near the Sun seems difficult to explain.

We now examine a second possibility. Fig. 13 shows the source surface extrapolation provided by the Wilcox Solar Observatory (WSO) indicating the HCS as extremely curved for Carrington rotation 2009 (23 October 2003-18 November 2003). It can be seen that the solar source is situated in the negative polarity of the coronal magnetic field. The HCS, $\sim 30^{\circ}$ away from the solar source, is strongly inclined to the ecliptic. The arrows in Fig. 13 indicate the orientation of the post-flare arcade from the southern (solid yellow) and the apex part of the filament (dashed yellow), as well as 


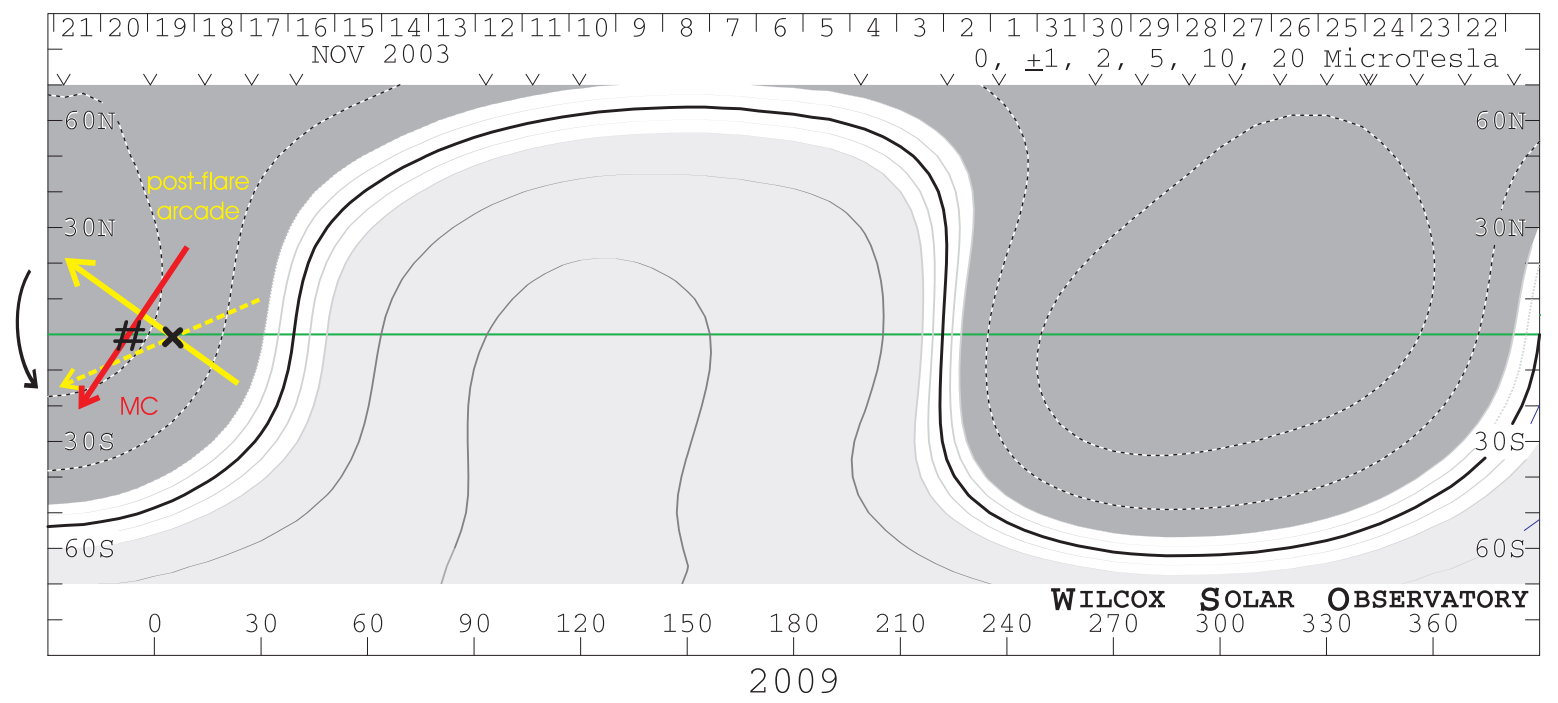

Fig. 13. WSO coronal magnetic field extrapolation at $2.5 \mathrm{R}_{\odot}$ of Carrington rotation 2009 showing the source surface field and the heliospheric current sheet (HCS). The position of the solar source and the associated MC that arrived at 20 Nov at the Earth are marked as $\mathbf{x}$ and \#, respectively. The orientation of the axial magnetic field of the post-flare arcade and the axial magnetic field of the MC with respect to the ecliptic are given as yellow and red arrows, respectively.

the orientation of the MC as measured in situ (solid red). At $1 \mathrm{AU}$, the MC is at the sector boundary, as the IMF before (after) the MC is pointing away (toward) the Sun. This can also be seen from Fig. 13 by tracing back the solar wind with constant velocity $(\sim 470 \mathrm{~km} / \mathrm{s})$ along the Parker spiral. We find that this solar wind has left the Sun $\sim 4$ days before, on 16 November 2003, which is at the location of the HCS. We conclude that the MC, propagating radially outward, overtook the solar wind in the IMF toward sector and ran into the HCS. It acts as a boundary for the ejected plasma and its shock which cannot be permeated (see Xie et al., 2006). Thus a rotation of the magnetic axis in order to align it with the HCS in IP space seems to be a plausible explanation for the change in the direction of the magnetic axis (cf. Smith, 2001; Yurchyshyn, 2007).

An alternative scenario is that from the AR a flux rope with negative twist started to rise and a right-handed MC was produced from the overlying coronal field through reconnection. Leamon et al. (2004) showed that the handedness in an AR does not necessarily imply the observation of a MC with the same handedness. A possible mechanism reversing the twist of a flux rope emerging into overlying pre-existing fields invoking reconnection is discussed in Zhang and Low (2003).

\section{Conclusions}

We have discussed the magnetic cloud on 20 November 2003 and its associated flare and CME with particular emphasis on the orientations and magnetic fluxes involved. From the available solar observations, we cannot determine the handedness of the erupting flux rope because the axial field di- rection is ambiguous. Assuming a right-handed flux rope erupting from low in the corona, a rotation of the axis would be necessary to match with the MC orientation. This may be explained through an interaction with the heliospheric current sheet during the propagation from Sun to Earth. Concerning the fluxes, the picture emerging from our case study is that the poloidal flux of the magnetic cloud $\Phi_{p}$ is in the same range as the reconnection flux $\Phi_{\bar{r}}$ of the flare, while the toroidal (axial) flux $\Phi_{t}$ of the MC is about one order of magnitude lower. This is in full agreement with studies by Qiu et al. (2007) and Longcope et al. (2007). This result gives us valuable insights to discriminate between the variety of CME-initiation models (see discussion in Qiu et al., 2007) and favors the in situ formation the flux ropes during the eruption (cf. Leamon et al., 2004), while being inline with the sheared arcade model by Démoulin et al. (1996). However, distinct uncertainties exist in calculating and comparing these fluxes, leaving the possibility that significant parts of the flux rope existed before the eruption. The sources of these uncertainties need to be carefully assessed. First, the flare reconnection flux depends on the chosen intensity threshold. Secondly, the loss of MC flux in interplanetary space can be substantial (50\%), and the length of the MC and the distribution of magnetic flux along the entire flux rope is hardly known. In this respect, upcoming coordinated STEREO-ACE/WIND and future HELEX observations will provide us with clues about the spatial structure of magnetic clouds, which is a necessary input for an accurate determination of both their fluxes as well as the helicity which they carry into interplanetary space. 
Acknowledgements. C. Möstl is supported by a research scholarship of the University of Graz and by the young researchers fund of the Steiermärkische Sparkasse. This work is supported by NASA/STEREO/PLASTIC grant to UNH, NASA/WIND/SWE grant and MFI grant NNG06GC75G, NNG06GD41G, and NNX08AD11G. We acknowledge funding by the Austrian Fonds zur Förderung der wissenschaftlichen Forschung under projects P20145-N16, P17100-N08, P15344 and by project J2512-N02 (E. Schrödinger Fellowship of M. Temmer). We thank P. Démoulin and G. Attrill for illuminating discussions and the referees for their very constructive comments.

Topical Editor R. Forsyth thanks V. Bothmer and V. Yurchyshyn for their help in evaluating this paper.

\section{References}

Attrill, G., Nakwacki, M. S., Harra, L. K., et al.: Using the Evolution of Coronal Dimming Regions to Probe the Global Magnetic Field Topology, Sol. Phys., 238, 117-139, doi:10.1007/ s11207-006-0167-5, 2006.

Bothmer, V.: Sources of magnetic helicity over the solar cycle, in: Solar Variability as an Input to the Earth's Environment, edited by: Wilson, A., vol. 535 of ESA Special Publication, pp. 419428, 2003.

Bothmer, V. and Schwenn, R.: The structure and origin of magnetic clouds in the solar wind, Ann. Geophys., 16, 1-24, 1998, http://www.ann-geophys.net/16/1/1998/.

Bothmer, V. and Zhukov, A.: The Sun as the prime source of space weather, in: Space Weather - Physics and Effects, edited by: Bothmer, V. and Daglis, I. A., Springer-Verlag, pp. 31-102, 2006.

Burlaga, L., Sittler, E., Mariani, F., and Schwenn, R.: Magnetic loop behind an interplanetary shock - Voyager, Helios, and IMP 8 observations, J. Geophys. Res., 86, 6673-6684, 1981.

Burlaga, L. F.: Magnetic clouds and force-free fields with constant alpha, J. Geophys. Res., 93, 7217-7224, 1988.

Chen, J., Howard, R. A., Brueckner, G. E., et al.: Evidence of an Erupting Magnetic Flux Rope: LASCO Coronal Mass Ejection of 1997 April 13, Astrophys. J. Lett., 490, L191, doi:10.1086/ 311029, 1997.

Cremades, H. and Bothmer, V.: On the three-dimensional configuration of coronal mass ejections, A\&A, 422, 307-322, doi: 10.1051/0004-6361:20035776, 2004.

Crooker, N. U.: Solar and heliospheric geoeffective disturbances, J. Atmos. Terr. Phys., 62, 1071-1085, 2000.

Crooker, N. U. and Horbury, T. S.: Solar Imprint on ICMEs, Their Magnetic Connectivity, and Heliospheric Evolution, Space Sci. Rev., 123, 93-109, doi:10.1007/s11214-006-9014-0, 2006.

Dasso, S., Mandrini, C. H., Démoulin, P., and Luoni, M. L.: A new model-independent method to compute magnetic helicity in magnetic clouds, A\&A, 455, 349-359, doi:10.1051/0004-6361: 20064806, 2006.

Dasso, S., Nakwacki, M. S., Démoulin, P., and Mandrini, C. H.: Progressive transformation of a flux rope to an ICME, Sol. Phys., 244, 115-137, 2007.

Démoulin, P.: Quantitative links between CMEs and magnetic clouds, Ann. Geophys., this issue, 2008.

Démoulin, P., Priest, E. R., and Lonie, D. P.: Three-dimensional magnetic reconnection without null points 2 . Application to twisted flux tubes, J. Geophys. Res., 101, 7631-7646, doi: 10.1029/95JA03558, 1996.

Fan, Y. and Gibson, S. E.: The Emergence of a Twisted Magnetic Flux Tube into a Preexisting Coronal Arcade, Astrophys. J. Lett., 589, L105-L108, doi:10.1086/375834, 2003.

Farrugia, C. J., Burlaga, L. F., Osherovich, V. A., et al.: A study of an expanding interplanetary magnetic cloud and its interaction with the earth's magnetosphere - The interplanetary aspect, J. Geophys. Res., 98, 7621-7632, 1993.

Farrugia, C. J., Vasquez, B., Richardson, I. G., et al.: A reconnection layer associated with a magnetic cloud, Adv. Space Res., 28, 759-764, 2001.

Farrugia, C. J., Popecki, M., Möbius, E., et al.: Wind and ACE observations during the great flow of 1-4 May 1998: Relation to solar activity and implications for the magnetosphere, J. Geophys. Res. (Space Physics), 107, 3-1, doi:10.1029/2001JA000188, 2002.

Farrugia, C. J., Matsui, H., Kucharek, H., et al.: Interplanetary coronal mass ejection and ambient interplanetary magnetic field correlations during the Sun-Earth connection events of October-November 2003, J. Geophys. Res. (Space Physics), 110, A09S13, doi:10.1029/2004JA010968, 2005.

Forbes, T. G. and Lin, J.: What can we learn about reconnection from coronal mass ejections, J. Atmos. Sol.-Terr. Phy., 62, 14991507, 2000.

Gonzalez, W. D. and Tsurutani, B. T.: Criteria of interplanetary parameters causing intense magnetic storms $\left(D_{s t}<-100 \mathrm{nT}\right)$, Planet. Space Sci., 35, 1101-1109, doi:10.1016/0032-0633(87) 90015-8, 1987.

Gopalswamy, N., Yashiro, S., Michalek, G., et al.: Solar source of the largest geomagnetic storm of cycle 23, Geophys. Res. Lett., 32, 12, doi:10.1029/2004GL021639, 2005.

Handy, B. N., Acton, L. W., Kankelborg, C. C., et al.: The transition region and coronal explorer, Sol. Phys., 187, 229-260, 1999.

Hasegawa, H., Sonnerup, B. U. Ö., Klecker, B., et al.: Optimal reconstruction of magnetopause structures from Cluster data, Ann. Geophys., 23, 973-982, 2005, http://www.ann-geophys.net/23/973/2005/.

Hasegawa, H., Sonnerup, B. U. Ö., Owen, C. J., et al.: The structure of flux transfer events recovered from Cluster data, Ann. Geophys., 24, 603-618, 2006, http://www.ann-geophys.net/24/603/2006/.

Hau, L.-N. and Sonnerup, B. U. Ö.: Two-dimensional coherent structures in the magnetopause: Recovery of static equilibria from single-spacecraft data, J. Geophys. Res., 104, 6899-6918, doi:10.1029/1999JA900002, 1999.

$\mathrm{Hu}$, Q. and Sonnerup, B. U. Ö.: Reconstruction of magnetic clouds in the solar wind: Orientations and configurations, J. Geophys. Res. (Space Physics), 107, 10-1, doi:10.1029/2001JA000293, 2002.

Hu, Q., Smith, C. W., Ness, N. F., and Skoug, R. M.: Multiple flux rope magnetic ejecta in the solar wind, J. Geophys. Res. (Space Physics), 109, 3102, doi:10.1029/2003JA010101, 2004.

Hudson, H. S.: Solar flares, microflares, nanoflares, and coronal heating, Sol. Phys., 133, 357-369, 1991.

Huttunen, K. E. J., Schwenn, R., Bothmer, V., and Koskinen, H. E. J.: Properties and geoeffectiveness of magnetic clouds in the rising, maximum and early declining phases of solar cycle 23, Ann. Geophys., 23, 625-641, 2005, 
http://www.ann-geophys.net/23/625/2005/.

Illing, R. M. E. and Hundhausen, A. J.: Observation of a coronal transient from 1.2 to 6 solar radii, J. Geophys. Res., 90, 275282,1985

Khrabrov, A. V. and Sonnerup, B. U. Ö.: deHoffmann-Teller analysis, in: Analysis Methods for Multi-Spacecraft Data, edited by: Paschmann, G. and Daly, P. W., (ESA SR-001; Noordwijk: ESA), p. 221, 1998.

Larson, D. E., Lin, R. P., McTiernan, J. M., et al.: Tracing the topology of the October 18-20, 1995, magnetic cloud with $\sim 0.1-10^{2} \mathrm{keV}$ electrons, Geophys. Res. Lett., 24, 1911-1914, doi:10.1029/97GL01878, 1997.

Leamon, R. J., Canfield, R. C., Jones, S. L., et al.: Helicity of magnetic clouds and their associated active regions, J. Geophys. Res. (Space Physics), 109, 5106, doi:10.1029/2003JA010324, 2004.

Lepping, R. P., Acuna, M. H., Burlaga, L. F., et al.: The Wind Magnetic Field Investigation, Space Sci. Rev., 71, 207-229, 1995.

Lin, R. P., Dennis, B. R., Hurford, G. J., et al.: The Reuven Ramaty High-Energy Solar Spectroscopic Imager (RHESSI), Sol. Phys., 210, 3-32, 2002.

Liu, Y., Richardson, J. D., Belcher, J. W., et al.: Constraints on the global structure of magnetic clouds: Transverse size and curvature, J. Geophys. Res. (Space Physics), 111, 12, doi: 10.1029/2006JA011890, 2006.

Longcope, D., Beveridge, C., Qiu, J., et al.: Modeling and Measuring the Flux Reconnected and Ejected by the Two-Ribbon Flare/CME Event on 7 November 2004, Sol. Phys., 244, 45-73, doi:10.1007/s11207-007-0330-7, 2007.

Lynch, B. J., Gruesbeck, J. R., Zurbuchen, T. H., and Antiochos, S. K.: Solar cycle-dependent helicity transport by magnetic clouds, J. Geophys. Res. (Space Physics), 110, 8107, doi: 10.1029/2005JA011137, 2005.

Manchester, W. B., Gombosi, T. I., Roussev, I., et al.: Threedimensional MHD simulation of a flux rope driven CME, J. Geophys. Res. (Space Physics), 109, 1102, doi:10.1029/ 2002JA009672, 2004.

Mandrini, C. H., Pohjolainen, S., Dasso, S., et al.: Interplanetary flux rope ejected from an X-ray bright point. The smallest magnetic cloud source-region ever observed, A\&A, 434, 725-740, doi:10.1051/0004-6361:20041079, 2005.

Marubashi, K.: Structure of the interplanetary magnetic clouds and their solar origins, Adv. Space Res., 6, 335-338, doi:10.1016/ 0273-1177(86)90172-9, 1986.

McComas, D. J., Gosling, J. T., Hammond, C. M., et al.: Magnetic reconnection ahead of a coronal mass ejection, Geophys. Res. Lett., 21, 1751, 1994.

McComas, D. J., Bame, S. J., Barker, P., et al.: Solar Wind Electron Proton Alpha Monitor (SWEPAM) for the Advanced Composition Explorer, Space Sci. Rev., 86, 563-612, doi:10.1023/A: $1005040232597,1998$.

Miklenic, C. H., Veronig, A. M., Vršnak, B., and Hanslmeier, A.: Reconnection and energy release rates in a two-ribbon flare, A\&A, 461, 697-706, doi:10.1051/0004-6361:20065751, 2007.

Mulligan, T., Russell, C. T., and Luhmann, J. G.: Solar cycle evolution of the structure of magnetic clouds in the inner heliosphere, Geophys. Res. Lett., 25, 2959-2962, doi:10.1029/98GL01302, 1998.

Ogilvie, K. W., Chornay, D. J., Fritzenreiter, R. J., et al.: SWE, A Comprehensive Plasma Instrument for the Wind Spacecraft,
Space Sci. Rev., 71, 55-77, 1995.

Qiu, J., Wang, H., Cheng, C. Z., and Gary, D. E.: Magnetic Reconnection and Mass Acceleration in Flare-Coronal Mass Ejection Events, Astrophys. J., 604, 900-905, doi:10.1086/382122, 2004.

Qiu, J., Hu, Q., Howard, T. A., and Yurchyshyn, V. B.: On the Magnetic Flux Budget in Low-Corona Magnetic Reconnection and Interplanetary Coronal Mass Ejections, Astrophys. J., 659, 758-772, doi:10.1086/512060, 2007.

Riley, P. and Crooker, N. U.: Kinematic Treatment of Coronal Mass Ejection Evolution in the Solar Wind, Astrophys. J., 600, 10351042, doi:10.1086/379974, 2004.

Russell, C. T. and Mulligan, T.: On the magnetosheath thicknesses of interplanetary coronal mass ejections, Planet. Space Sci., 50 , 527-534, 2002.

Rust, D. M. and LaBonte, B. J.: Observational Evidence of the Kink Instability in Solar Filament Eruptions and Sigmoids, Astrophys. J. Lett., 622, L69-L72, doi:10.1086/429379, 2005.

Scherrer, P. H., Bogart, R. S., Bush, R. I., and MDI Engineering Team: The Solar Oscillations Investigation - Michelson Doppler Imager, Sol. Phys., 162, 129-188, 1995.

Schmidt, J. M. and Cargill, P. J.: Magnetic reconnection between a magnetic cloud and the solar wind magnetic field, J. Geophys. Res. (Space Physics), 108, 5-1, doi:10.1029/2002JA009325, 2003.

Shodhan, S., Crooker, N. U., Kahler, S. W., et al., J. T.: Counterstreaming electrons in magnetic clouds, J. Geophys. Res., 105, 27 261-27 268, doi:10.1029/2000JA000060, 2000.

Siscoe, G. and Schwenn, R.: CME Disturbance Forecasting, Space Sci. Rev., 123, 453-470, doi:10.1007/s11214-006-9024-y, 2006.

Smith, C. W., L'Heureux, J., Ness, N. F., et al.: The ACE Magnetic Fields Experiment, Space Sci. Rev., 86, 613-632, doi:10.1023/A: 1005092216668, 1998.

Smith, E. J.: The heliospheric current sheet, J. Geophys. Res., 106, 15 819-15 832, doi:10.1029/2000JA000120, 2001.

Sonnerup, B. U. Ö., Hasegawa, H., and Paschmann, G.: Anatomy of a flux transfer event seen by Cluster, Geophys. Res. Lett., 31, 11 803, doi:10.1029/2004GL020134, 2004.

Xie, Y., Wei, F., Xiang, C., and Feng, X.: The Effect of the Heliospheric Current Sheet on Interplanetary Shocks, Sol. Phys., 238, 377-390, doi:10.1007/s11207-006-0227-x, 2006.

Yurchyshyn, V.: Relationship between EIT Posteruption Arcades, Coronal Mass Ejections, the Coronal Neutral Line, and Magnetic Clouds, Astrophys. J. Lett., 675, L49-L52, 2008.

Yurchyshyn, V., Hu, Q., and Abramenko, V.: Structure of magnetic fields in NOAA active regions 0486 and 0501 and in the associated interplanetary ejecta, Space Weather, 3, 8, doi: 10.1029/2004SW000124, 2005.

Yurchyshyn, V., Liu, C., Abramenko, V., and Krall, J.: The May 13, 2005 Eruption: Observations, Data Analysis and Interpretation, Sol. Phys., 239, 317-335, doi:10.1007/s11207-006-0177-3, 2006.

Yurchyshyn, V., Hu, Q., Lepping, R. P., Lynch, B. J., and Krall, J.: Orientations of LASCO Halo CMEs and Their Connection to the Flux Rope Structure of Interplanetary CMEs, Adv. Space Res., 40, 1821-1826., 2007.

Yurchyshyn, V. B., Wang, H., Goode, P. R., and Deng, Y.: Orientation of the Magnetic Fields in Interplanetary Flux Ropes and Solar Filaments, Astrophys. J., 563, 381-388, doi:10.1086/323778, 2001. 
Zhang, M. and Low, B. C.: Magnetic Flux Emergence into the Solar Corona. III. The Role of Magnetic Helicity Conservation, Astrophys. J., 584, 479-496, doi:10.1086/345615, 2003.
Zhao, X. P. and Hoeksema, J. T.: Central axial field direction in magnetic clouds and its relation to southward interplanetary magnetic field events and dependence on disappearing solar filaments, J. Geophys. Res., 103, 2077-2083, 1998. 